\title{
Craniopharyngeal Canal and Its Spectrum of Pathology
}

T.A. Abele, K.L. Salzman, H.R. Harnsberger, and C.M. Glastonbury

\begin{abstract}
BACKGROUND AND PURPOSE: The craniopharyngeal canal is a rare, well-corticated defect through the midline of the sphenoid bone from the sellar floor to the anterosuperior nasopharyngeal roof. We reviewed a series of craniopharyngeal canals to determine a system of classification that might better our understanding of this entity, highlight the range of associated pathologic conditions, and optimize patient treatment.
\end{abstract}

MATERIALS AND METHODS: Available MR imaging, CT, and clinical data (from 1989-2013) of 29 patients (10 female, 15 male, 4 unknown; median age, 4 years; age range, 1 day-65 years) with craniopharyngeal canals were retrospectively examined. Qualitative assessment included orthotopic or ectopic adenohypophysis and the presence of a tumor and/or cephalocele. The midpoint anteroposterior diameter was measured. Clinical and imaging data were evaluated for pituitary dysfunction and accompanying anomalies.

RESULTS: Craniopharyngeal canals were qualitatively separated into 3 types: incidental canals (type 1); canals with ectopic adenohypophysis (type 2); and canals containing cephaloceles (type 3A), tumors (type 3B), or both (type 3C), including pituitary adenoma, craniopharyngioma, dermoid, teratoma, and glioma. Quantitative evaluation showed a significant difference $(P<.0001)$ in the anteroposterior diameters of type 1 canals (median, 0.8; range, 0.7-1.1 mm), type 2 canals (median, 3.9, range, 3.5-4.4 mm), and type 3 canals (median, 9.0; range, 5.9-31.0 mm) imparting small, medium, and large descriptors. Canals with cephaloceles all contained an ectopic adenohypophysis. The craniopharyngeal canals were associated with pituitary dysfunction (6/29) and congenital anomalies (8/29).

CONCLUSIONS: Accurate diagnosis and classification of craniopharyngeal canals are valuable to characterize lesions requiring surgery, identify patients with potential pituitary dysfunction, and avoid iatrogenic hypopituitarism or CSF leak during surgical resection of nasopharyngeal masses.

ABBREVIATIONS: $\mathrm{AP}=$ anteroposterior; $\mathrm{CPC}=$ craniopharyngeal canal

$\mathrm{T}$ he craniopharyngeal canal (CPC) is a rare, well-corticated defect of the midline sphenoid body from the sellar floor to the nasopharynx. Although embryogenesis of the canal has been disputed, evidence supports its origin from incomplete closure of the Rathke pouch, the precursor of the adenohypophysis. ${ }^{1-3}$ Since the arrival of modern cross-sectional imaging, the literature on CPCs has been limited to case reports and a few small case series, which

Received June 27, 2013; accepted after revision August 15.

From the Department of Radiology (T.A.A., K.L.S., H.R.H.), University of Utah, Salt Lake City, Utah; and Department of Radiology (C.M.G.) University of California, San Francisco, San Francisco, California.

Paper previously presented at: Annual Meeting of the American Society of Neuroradiology, May 23, 2013; San Diego, California.

Please address correspondence to Travis A. Abele, MD, Department of Radiology, University of Utah, 30 North 1900 East \#1A071, Salt Lake City, UT 84132; e-mail: travis.abele@hsc.utah.edu

三 Indicates article with supplemental on-line tables.

http://dx.doi.org/10.3174/ajnr.A3745 describe variably sized canals and a range of associated lesions. ${ }^{2,4-13} \mathrm{CPC}$ is a rare but important entity to recognize in the evaluation of nasopharyngeal or midline skull base lesions. Correct diagnosis may indicate pituitary dysfunction, obviate the need for surgery, and prevent iatrogenic hypopituitarism or CSF leak.

CPCs have previously been described as either small ${ }^{14}(<1.5$ $\mathrm{mm}$ diameter) or large ${ }^{4}$ with or without mass; however, this binary division does not fully characterize its range. In this retrospective review of 29 patients with CPCs, we use quantitative and qualitative imaging features to provide a new system of classification, which divides the malformation into 3 types that more accurately describe size and associated pathologic appearance: small incidental canals (type 1); medium-sized canals containing ectopic (inferiorly displaced) adenohypophysis (type 2); and large canals with associated cephaloceles (type $3 \mathrm{~A}$ ), tumors of the adenohypophysis and associated embryonic tissues (type $3 \mathrm{~B}$ ), or both (type 3C). In addition, we examine the embryology and relevant 

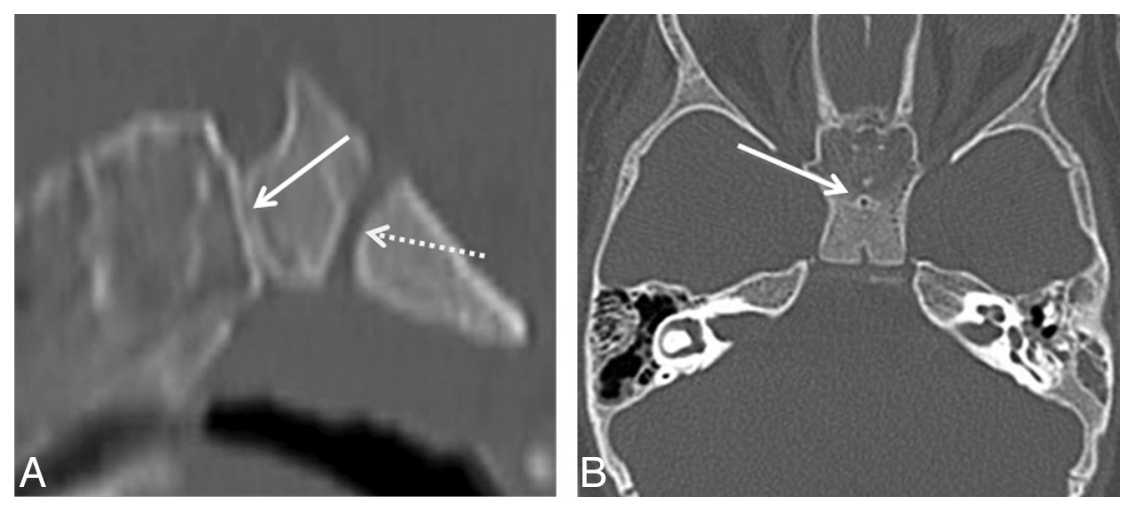

symptoms of pituitary dysfunction were presumed to have normal pituitary function. All tumors were resected, and diagnosis was based on pathologic analysis.

\section{RESULTS}

Twenty-nine patients (age range, 1 day-65 years; median age, 4 years) had radiologically identified CPCs on CT (3 patients), MR imaging (15 patients), or both CT and MR imaging (11 patients). Ten patients were female, 15 male, and in 4 patients, sex was not provided in available clinical data. Of 29 patients, 9 (31\%) had type 1 incidental canals with orthotopic adenohypophysis and no cephalocele or tumor. Type 2 CPCs with ectopic adenohypophysis and no associated cephliterature of the CPC to refine understanding of this rare entity and its associated congenital anomalies and neoplasms.

\section{MATERIALS AND METHODS}

In our study, which was approved by our institutional review board and is compliant with the Health Insurance Portability and Accountability Act, 2 neuroradiologists with Certificates of Added Qualification and a neuroradiology fellow retrospectively examined available clinical and imaging data of a series of radiologically proven CPCs reviewed or treated at the University of Utah during the past 25 years (1989-2013). Radiologic diagnosis of CPC was defined as a bony channel extending from the floor of the sella turcica to the inferior border of the sphenoid body. All patients underwent cross-sectional imaging studies: CT, MR imaging, or both.

Qualitative imaging evaluation was based on 2 criteria: 1) orthotopic or ectopic position of the adenohypophysis, and 2) cephalocele and/or neoplasm within the canal. These criteria were then used to divide the CPCs into 3 types: incidental canals with orthotopic adenohypophysis and no cephalocele or tumor (type 1); canals with ectopic (inferiorly displaced) adenohypophysis but no associated cephalocele or tumor (type 2); and canals with associated cephalocele (type 3A), tumor (type 3B), or both (type 3C). Neurohypophysis location was documented when identified. Cephalocele was defined as herniation of the meninges and CSF contents into the CPC with or without herniation of the pituitary gland and its associated structures.

We performed quantitative evaluation by measuring the anteroposterior (AP) diameter at the midpoint of the CPC from the anterior to posterior wall of the canal on a sagittal MR imaging sequence or CT reconstruction. When a neoplasm obliterated the walls of the CPC, the anterior and posterior margins of the tumor were used as a surrogate measure of the canal diameter. All measurement data were expressed as median and range. One-way ANOVA was used to assess statistically significant difference among AP diameter measurements of the 3 qualitative types of CPCs.

Clinical information was compiled via a retrospective chart review and included available demographic information, presenting symptoms, the presence of pituitary dysfunction, pathologic features, or the presence of other congenital anomalies. Patients without

alocele or tumor were seen in 7 (24\%) of 29 patients. Forty-five percent $(13 / 29)$ of patients had type 3 CPCs associated with cephaloceles $(4 / 29$, type $3 \mathrm{~A})$, tumors $(7 / 29$, type $3 \mathrm{~B})$, or both $(2 / 29$, type $3 \mathrm{C}$ ) including pituitary adenoma, craniopharyngioma, dermoid, teratoma, and glioma.

The AP diameter of each qualitative CPC type were as follows: type 1 canals: median, 0.8 ; range, $0.7-1.1 \mathrm{~mm}$; type 2 canals: median, 3.9; range, 3.5-4.4 mm; and type 3 canals: median, 9.0; range, 5.9-31.0 mm. ANOVA analysis demonstrated statistically significant difference $(P<.0001)$ among the AP diameter measurements of each type of CPC, thus imparting size descriptors: small, medium, and large. There was no significant difference between the AP diameters of the type $3 \mathrm{~A}, 3 \mathrm{~B}$, and $3 \mathrm{C}$ canals.

Pituitary dysfunction was documented in $6(21 \%)$ of all 29 CPCs and in $6(30 \%)$ of the 20 type 2 and type 3 canals. Hormone abnormalities included generalized hypopituitarism (2 patients), partial hypopituitarism, diabetes insipidus, and growth hormone deficiency ( 2 patients). Other congenital anomalies were seen in 8 of 29 patients with no predilection for type of CPC. Patient data are summarized in On-line Table 1, and complete patient data are provided in On-line Table 2.

Type 1 small, incidental CPCs with orthotopic adenohypophysis (Fig 1) demonstrated a well-corticated channel on CT extending from the inferior floor of the sella turcica to the inferior border of the midline sphenoid body and measured $\geq 1.1 \mathrm{~mm}$ in AP diameter. On MR imaging, they showed the same midline path through the sphenoid bone with variable internal signal. These patients underwent imaging for symptoms unrelated to the CPC, such as sinus disease and trauma; however, some patients were known to have congenital anomalies including PHACE (posterior fossa malformations, hemangiomas, arterial abnormalities, cardiac defects, eye abnormalities) syndrome, ${ }^{15}$ Blake remnant, duplicated pituitary gland, and congenital cleft lip or cleft palate. The patient with the duplicated pituitary gland also had a nasopharyngeal teratoma, which did not involve the CPC. None of the patients with type 1 CPCs had pituitary dysfunction or contained ectopic pituitary tissue, cephalocele, or tumor.

Type 2 medium-sized CPCs presented as well-corticated channels (3.5-4.4 mm AP diameter) on CT and, on MR imaging, 


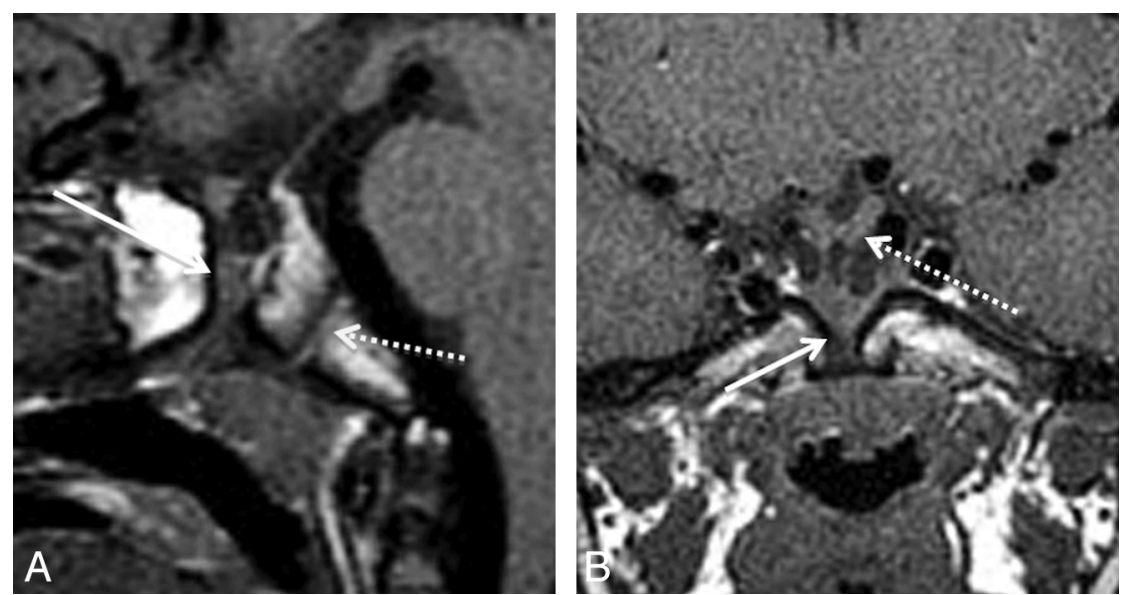

FIG 2. A 4-year-old boy with hypopituitarism and a type 2 CPC. A, Sagittal T1 MR image shows a medium-sized canal with ectopic, inferiorly displaced pituitary tissue (arrow). The AP diameter of this canal measured $3.5 \mathrm{~mm}$. Note the normal spheno-occipital synchondrosis (dashed arrow). B, Coronal T1 MR image demonstrates soft tissue within the CPC (arrow). There is mild inferior displacement of the optic chiasm (dashed arrow) and infundibular recess of the third ventricle.
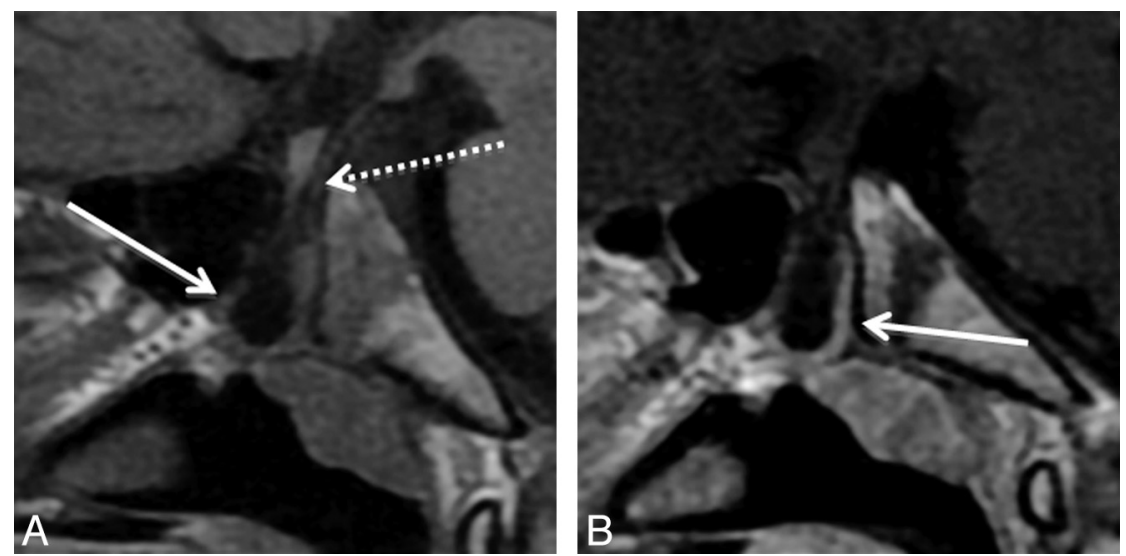

FIG 3. A 10-year-old boy with growth hormone deficiency and short stature with a type $3 A$ CPC. $A$, Sagittal T1 MR image shows a large CPC containing a cephalocele with herniated adenohypophysis (arrow) and CSF. Note displacement of the infundibular recess of the third ventricle (dashed arrow). B, Sagittal TI postcontrast MR image shows enhancement of the adenohypophysis (arrow), which, in our 4 type 3A canals, was invariably positioned along the posterior and/or inferior aspect of the cephalocele.

contained inferiorly located ectopic adenohypophysis (Fig 2). Adenohypophysis was identified and was distinguished from tumor on MR imaging by a characteristic homogeneous $\mathrm{T} 1$ signal isointense to the brain and homogeneous enhancement. Other characteristic features were the location of the dorsal T1 hyperintense neurohypophysis and infundibular stalk extending to the superior surface of the adenohypophysis. Of 7 patients, 4 also had inferiorly located neurohypophysis. One medium-sized canal had orthotopic neurohypophysis, and in another case, the neurohypophysis was not identified. One type $2 \mathrm{CPC}$ was partially patent on sagittal T1-weighted MR imaging and had suprasellar ectopic posterior pituitary tissue inferior to the hypothalamus. Type 2 CPCs were found in patients who clinically presented with pituitary dysfunction (2/7), headache, coloboma, and hydrocephalus. The patient with hydrocephalus had congenital abnormalities, including cortical dysplasia, absent right ICA, and schizencephaly. Other congenital anomalies included holoprosencephaly and coloboma.
The 4 type 3A large CPCs containing only cephalocele demonstrated well-corticated channels on CT that, on MR imaging, contained CSF; meninges; ectopic adenohypophysis (4/4); the pituitary infundibulum; infundibular recess of the third ventricle; and, in 1 case, an associated arachnoid cyst (Fig 3). The 4 patients with type 3A CPCs presented with growth hormone deficiency, generalized hypopituitarism, diabetes insipidus, or meningitis. These patients had no other documented congenital anomalies.

Type 3B large CPCs containing tumors consisted of 2 gliomas, 1 dermoid, 1 teratoma, 1 craniopharyngioma, and 2 adenomas. Type 3C large CPCs containing tumor and cephalocele consisted of 2 dermoids (Fig 4). Most tumors had a large portion of their mass within the CPC. One of the nasopharyngeal gliomas extended intracranially into the sella turcica, and the other glioma passed superiorly through the canal into the suprasellar space (Fig $4 B$ ). In both type $3 \mathrm{C}$ and 2 type 3B CPCs, there was ectopic adenohypophysis. In the cases of craniopharyngioma and adenoma, which obliterated the canal walls, the adenohypophysis was orthotopic. Of the 9 type $3 \mathrm{~B}$ and type $3 \mathrm{C} C \mathrm{CP}$, 4 presented as a nasopharyngeal mass, and 1 presented with growth hormone deficiency. The 1 patient with teratoma had a duplicated pituitary gland. Otherwise, there were no documented associated congenital anomalies in type 3 large canals.

\section{DISCUSSION}

The CPC is a rare, well-corticated defect of the midline sphenoid body extending from the floor of the sella turcica to the roof of the nasopharynx. ${ }^{14}$ To our knowledge, our study is the largest series of CPCs since the advent of cross-sectional imaging. On the basis of quantitative and qualitative features, CPCs can be classified into 3 types: small incidental canals (type 1); medium-sized canals with ectopic pituitary tissue (type 2); and large canals containing cephaloceles (type 3A), tumors (type 3B), or both (type 3C). Identification and classification of CPCs have prognostic and therapeutic implications. Type 1 small incidental canals are benign but may be found in patients with other craniofacial or neural congenital anomalies. Type 2 medium-sized canals and type $3 \mathrm{~A}$ large canals with cephaloceles are associated with ectopic adenohypophysis and possible pituitary dysfunction. Type 3 large CPCs containing tumors and/or cephaloceles often contain ectopic pituitary tissue within the nasopharynx; thus, care should be taken during surgery to avoid iatrogenic hypopituitarism and/or CSF leak. Compared with the previous division of small benign $\mathrm{CPCs}^{14}$ or large $\mathrm{CPCs}$ 


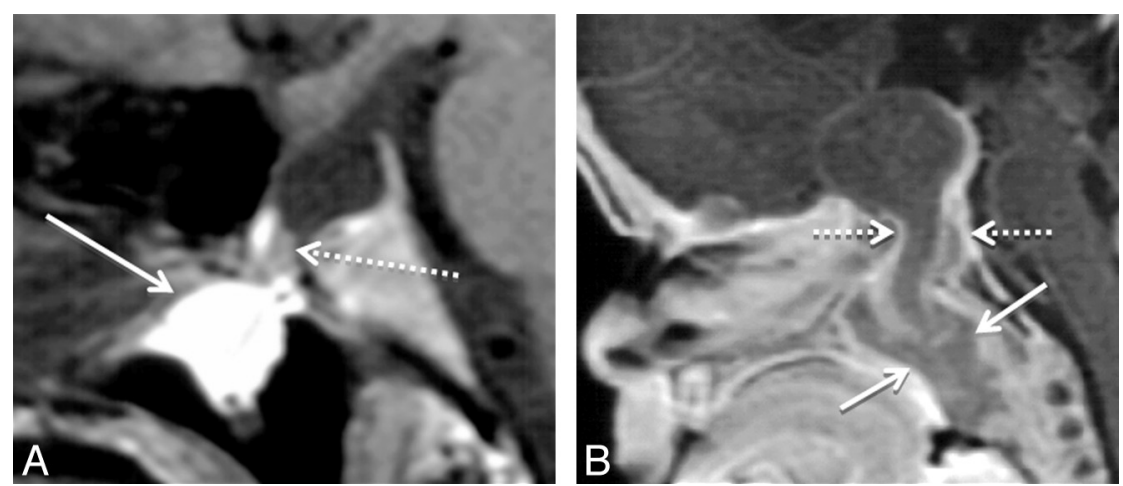

FIG 4. Two different patients with type 3 large CPCs containing tumor. A, Sagittal T1 MR image shows a large CPC containing portions of a T1 hyperintense dermoid (arrow) in a 30-year-old woman who presented with a nasopharyngeal mass. Note the associated cephalocele with herniated CSF and the adenohypophysis (dashed arrow). This is a type 3B CPC. $B$, Sagittal T1 postcontrast MR image in an 8-week-old girl who underwent imaging for a nasopharyngeal mass demonstrates a nasopharyngeal glioma (arrows), with mild enhancement extending into the suprasellar space through a large CPC (dashed arrows). This is a type 3C CPC.
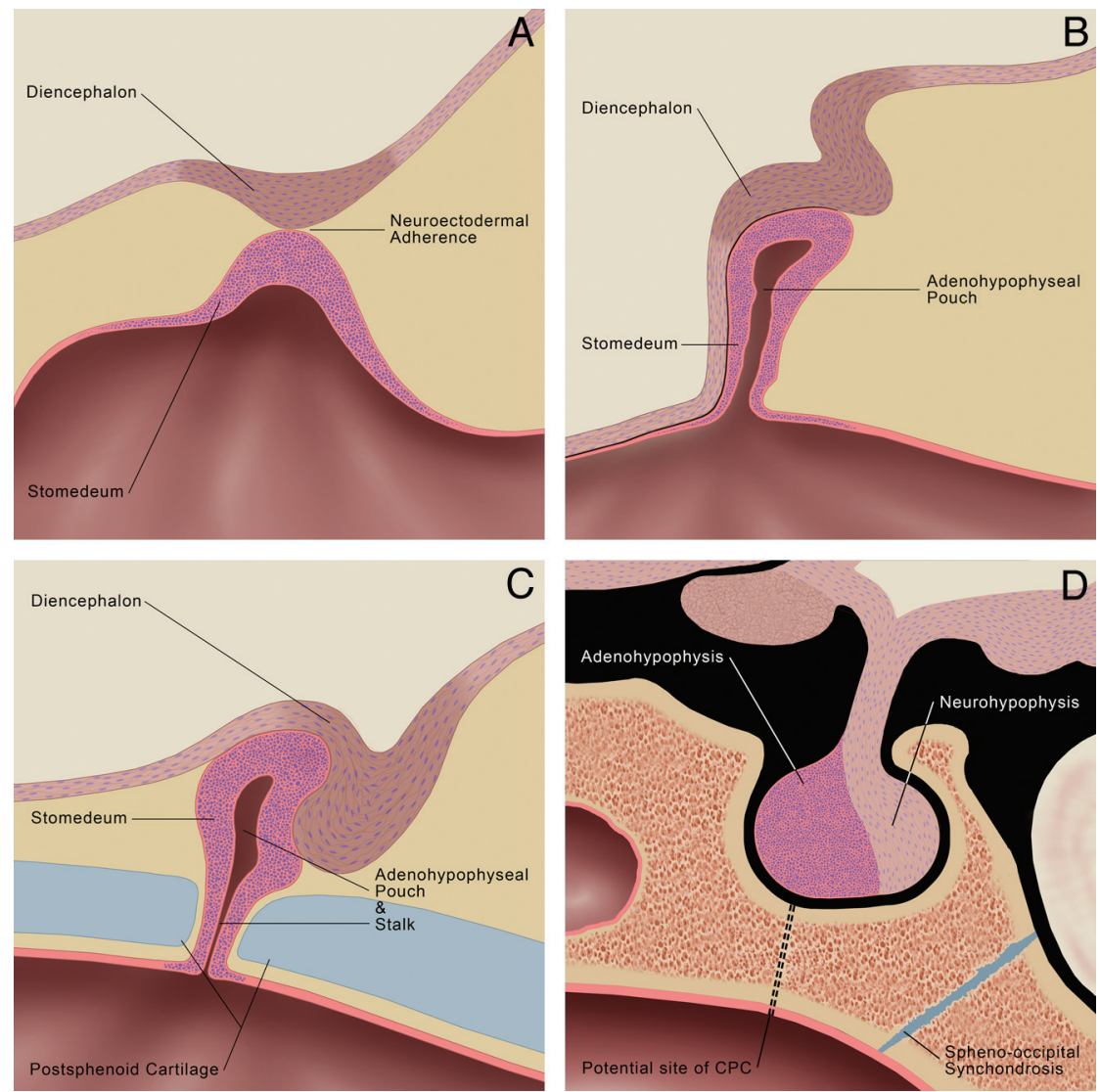

FIG 5. Embryology of the normal pituitary gland. A, At 3-4 weeks of gestation, the neuroectodermal adherence develops at a point of contact between the diencephalon (neurohypophyseal anlage) and roof of the stomodeum (adenohypophyseal anlage). B, At 4-5 weeks, the neuroectodermal adherence migrates dorsally forming the adenohypophyseal (Rathke) pouch, while the diencephalon migrates dorsal to the stomodeum to assume the position of the future neurohypophysis. The adenohypophyseal pouch elongates forming a stalk at its ventral aspect at 5-6 weeks (not shown). C, At 6-7 weeks, the postsphenoid cartilage develops, which results in obliteration of the adenohypophyseal stalk. $D$, In this illustration of the infant pituitary gland and sella, a dotted line traces the path of the potential CPC, which is believed to arise from nonobliteration of the adenohypophyseal stalk. The spheno-occipital synchondrosis (illustrated in blue) does not close until approximately age 16 years and mimics the CPC in children. Graphic illustrations used with permission from Amirsys. with associated nasopharyngeal masses and craniofacial malformations, ${ }^{4}$ our classification provides a more nuanced characterization of the CPC with the inclusion of medium-sized type 2 canals and subdivision of large type 3 canals.

From our data, a nasopharyngeal mass should raise the suspicion for a type 3 $\mathrm{CPC}$, and pituitary dysfunction may suggest a type 2 or 3 CPC. CT is sufficient to characterize type $1 \mathrm{CPCs}$, unless there is suspicion for associated craniofacial congenital anomalies. MR imaging, however, is the best for evaluation of ectopic adenohypophysis in type 2 CPCs and cephalocele and/or tumor in type 3 CPCs due to its superior contrast resolution.

The CPC is postulated to arise from an error in the normal development of the pituitary gland (Fig 5). The pituitary gland originates from a single embryologic neuroectodermal adherence formed by fusion of the roof of the stomodeum (adenohypophysis anlage) and floor of the diencephalon (neurohypophysis anlage). ${ }^{16}$ At approximately the fourth week of gestation, the forebrain grows rostrocaudally and mesoderm proliferates ventrolaterally around the neuroectodermal adherence, causing the adherence to migrate dorsally and form an invagination, called the adenohypophyseal (Rathke) pouch. ${ }^{16,17}$ During the fifth and sixth weeks of gestation, the adenohypophyseal pouch elongates, forming a narrow stalk between it and the stomodeum. ${ }^{18}$ At approximately the sixth to seventh weeks of gestation, the cartilaginous sphenoid skull base develops, obliterating this adenohypophyseal stalk. ${ }^{18,19}$ The sphenoid cartilage is composed of 2 paired presphenoid and 2 paired postsphenoid cartilages. The presphenoid cartilages fuse and later form the anterior sphenoid body, and the postsphenoid cartilages fuse to form the basisphenoid cartilage, which later becomes the posterior sphenoid body and sella turcica. ${ }^{20}$ Defective fusion of the postsphenoid cartilages results in nonobliteration of the stalk between the adenohypophyseal pouch and the stomodeum and a residual canal extending from the sella turcica to the pharynx, named the CPC.

Two case series from the mid-20th century proposed that the CPC is merely a remnant of a vascular channel. Arey ${ }^{14}$ 
came to this conclusion when, after examining the sphenoid bone of 17 embryonic specimens for persistence of a CPC containing pituitary tissue, he only found channels for blood vessels. Lowman et $\mathrm{al}^{21}$ radiographically identified 2 complete CPCs in 400 neonate sphenoid bone specimens, but when he histologically analyzed the specimens, he found only vascular channels without evidence of "squamous or ciliated epithelium." Both authors erroneously concluded that the absence of evidence for the pituitary origin of the CPC suggests that it does not arise from the embryologic pituitary.

Subsequent studies make a strong case for the pituitary origin. Kjaer and Fischer-Hansen ${ }^{1}$ used modern immunohistochemistry to demonstrate adenohypophyseal tissue within the CPC of $6 \mathrm{fe}-$ tuses with holoprosencephaly or anencephaly. Moreover, Kjaer and Fischer-Hansen noted that the postsphenoid bone is nearly always malformed in holoprosencephaly and invariably malformed in anencephaly, supporting an association between malformation of the cartilaginous sphenoid skull base and persistence of the CPC. Hori et $\mathrm{al}^{3}$ evaluated nasopharyngeal pituitary tissue of fetal specimens and demonstrated adenohypophyseal tissue in a CPC. These authors also performed a meta-analysis showing that 24 of 27 ectopic pituitary adenomas in the literature occurred in either the sphenoid sinus or the clivus. Hori et $\mathrm{al}^{3}$ believed this evidence for the persistence of pharyngeal pituitary tissue into adulthood; however, one could more convincingly propose, given the nonaggressive nature of pituitary adenomas, that the bony location of these ectopic adenomas supports pituitary tissue within a CPC rather than in the pharynx. The same conclusion can be made from the craniopharyngioma and adenomas in our series and case reports of ectopic intrasphenoid pituitary neoplasms in the literature. ${ }^{2,12,13}$

The characteristics of our type 1 small incidental CPCs are similar to those of the meta-analysis performed by Arey. ${ }^{14}$ Of 8338 skulls in 11 studies, 35 CPCs were identified, yielding a prevalence of $0.42 \%$. Each canal extended from the sella turcica to the basal surface of the sphenoid bone, was surrounded by "compact" bone, and measured $<1.5 \mathrm{~mm}$. These features are comparable to our type 1 canals, which were delimited by cortical bone and measured 0.7-1.1 mm in AP diameter. Small CPCs were first systematically evaluated by Landzert (who coined the term craniopharyngeal canal) in 1868 . He identified a complete bony sphenoid canal the width of a bristle in $10(10 \%)$ of 100 anatomic specimens from infants up to 8 months old. ${ }^{14}$ A study in 1903 showed similar prevalence $(18 / 200)$ in infants younger than 3 months old. ${ }^{14}$ Comparing these data with Arey's frequency of $0.42 \%$ raises the question of whether some CPCs obliterate in early childhood. To our knowledge, no studies have investigated this matter.

An in vivo type 2 medium-sized CPC with ectopic pituitary tissue was first reported in 1949 by Wilkerson and Cayce, ${ }^{22}$ in which a 19-year-old woman had a 3-5 $\mathrm{mm}$ diameter canal at the base of the sphenoid discovered during choanal atresia surgery. The canal was radiographed during extensive probing, and the patient subsequently experienced hypothyroidism and secondary amenorrhea. This development is not surprising given the presence of ectopic adenohypophysis in all type 2 CPCs in our series. Two recent case reports discuss medium-sized CPCs with ectopic pituitary tissue. One had an associated hypothalamic hamartoma, measured $>1.5 \mathrm{~mm}$ in diameter (exact measurement not provided), and had inferior ectopic adenohypophysis on MR imaging. ${ }^{11}$ Currarino et $\mathrm{al}^{4}$ reported the other medium-sized CPC in their article on "large craniopharyngeal canals." One of their 2 cases described a male infant with a 3-mm diameter CPC containing mildly displaced adenohypophysis (as demonstrated in the figures) but no pituitary dysfunction. This patient also had congenital anomalies, including cleft lip and palate, hypertelorism, and orbital hypoplasia. Type 2 CPCs are important to recognize not only for their apparent association with congenital abnormalities but also for the detection of related pituitary dysfunction. In our series, 2 of 7 type 2 CPCs had pituitary dysfunction. We postulate the cause to be ectopic pituitary tissue, as our type $3 \mathrm{~A}$ large CPCs with cephaloceles and more displaced pituitary tissue had a higher rate of pituitary deficiency (4/6) than the type 2 canals.

A case of type 3A large CPC containing cephalocele and ectopic pituitary tissue was first described by Klinkosch in 1764, in which a neonate had a 5 - $\mathrm{mm}$ wide passage extending through the middle of the sphenoid that contained prolapsed dura and an ectopic pituitary gland. ${ }^{14}$ Recent case reports of large CPCs with cephaloceles containing ectopic pituitary tissue describe canals measuring $10-13 \mathrm{~mm}$ in diameter. ${ }^{8,9,23,24}$ One patient presented with recurrent meningitis (as in our series), and 2 patients had colobomas, believed to result from stretching of the optic nerves into the cephalocele. ${ }^{23-25}$ Three of 4 large CPCs in the literature that were identified before surgery had pituitary dysfunction, reflecting our $75 \%$ prevalence in large canals. ${ }^{9,23}$ Hughes et $\mathrm{al}^{8}$ described 2 patients with large CPCs and cephaloceles who presented with suspected iatrogenic hypopituitarism after resection of nasopharyngeal "masses." This finding underscores the importance of recognizing cephaloceles and ectopic pituitary tissue in type 3A CPCs to avoid CSF leak and/or pituitary resection during nasopharyngeal mass surgery.

Fewer than 25 cases of ectopic pituitary adenomas are reported within the sphenoid sinus, presumed to originate within type $3 \mathrm{~B}$ CPCs. Most of these lesions presented due to mass effect (as in 1 of our 2 cases) or ectopic hormone secretion. ${ }^{2,26}$ Reports of infrasellar craniopharyngiomas are rarer, with at least 5 reported in the modern literature. ${ }^{13,27-30}$ An infrasellar location separate from the sella turcica is important to recognize because it indicates that the craniopharyngioma originated within a type $3 \mathrm{~B}$ CPC rather than invading the skull base inferiorly from a sellar origin.

Dermoids, teratomas, and gliomas are uncommon lesions of the nasopharynx. ${ }^{31,32}$ Nasopharyngeal teratoma can be associated with duplication of the adenohypophysis, as in our case. ${ }^{33}$ The epignathus type of teratoma is a large neoplasm that fills the oral cavity and has been reported in association with a CPC. ${ }^{5}$ Nasopharyngeal glioma is a rare entity composed of heterotopic neuroglial tissue. Although there are reported cases of nasopharyngeal gliomas associated with midline skull base defects, none are explicitly associated with CPCs or extend intracranially as our cases did. ${ }^{32}$ As in type 3 CPCs with cephalocele, identifying intracranial extension of a dermoid, teratoma, or glioma into a type 3 CPC is important for surgical planning to avoid inadvertent CSF leak and potential meningitis.

Limitations of this series were its relatively small size and retrospective nature. In addition, because many of the cases were 
referred for evaluation from outside institutions, we had no control of the quality or protocol of the imaging examinations and completeness of the clinical data.

\section{CONCLUSIONS}

CPCs can be classified into 3 types, which have therapeutic and prognostic implications: small incidental canals (type 1); medium-sized canals with ectopic adenohypophysis (type 2); and large canals containing cephaloceles (type 3A), tumors (type 3B), or both (type 3C). Type 2 CPCs and type 3A large canals with cephaloceles have ectopic adenohypophysis and should alert the clinician to possible pituitary dysfunction. Type 3 large CPCs associated with tumors and/or cephaloceles often contain ectopic pituitary tissue within the nasopharynx or have continuity with the dural meninges; thus, care should be taken during surgery to avoid iatrogenic hypopituitarism and/or CSF leak.

Disclosures: Karen L. Salzman—UNRELATED: Consultancy: Amirsys Publishing; Royalties: Amirsys Publishing. H. Ric Harnsberger-Employment: Amirsys; Royalties: Amirsys; Stock/Stock Options: Amirsys. Christine M. Glastonbury-UNRELATED: Consultancy: Amirsys; Royalties: Amirsys; Stock/Stock Options: Amirsys.

\section{REFERENCES}

1. Kjaer I, Fischer-Hansen B. Human fetal pituitary gland in holoprosencephaly and anencephaly. J Craniofac Genet Dev Biol 1995;15: 222-29

2. Kaushik C, Ramakrishnaiah R, Angtuaco EJ. Ectopic pituitary adenoma in persistent craniopharyngeal canal: case report and literature review. J Comput Assist Tomogr 2010;34:612-14

3. Hori A, Schmidt D, Rickels E. Pharyngeal pituitary: development, malformation, and tumorigenesis. Acta Neuropathol 1999;98: 262-72

4. Currarino G, Maravilla KR, Salyer KE. Transsphenoidal canal (large craniopharyngeal canal) and its pathologic implications. AJNR Am J Neuroradiol 1985;6:39-43

5. Cohen R, Nelson MD, Segall HD. Epignathic teratoma associated with craniopharyngeal canal. AJNR Am J Neuroradiol 1989;10:652

6. Rottenberg GT, Chong WK, Powell M, et al. Cyst formation of the craniopharyngeal duct. Clinical Radiol 1994;49:126-29

7. Kjaer I, Russell BG. The craniopharyngeal canal indicating the presence of pharyngeal adenopituitary tissue. Eur J Radiol 1995;20: 212-14

8. Hughes ML, Carty AT, White FE. Persistent hypophyseal (craniopharyngeal) canal. Br J Radiol 1999;72:204-06

9. Ekinci G, Kiliç T, Baltacioğlu F, et al. Transsphenoidal (large craniopharyngeal) canal associated with a normally functioning pituitary gland and nasopharyngeal extension, hyperprolactinemia, and hypothalamic hamartoma. AJR Am J Roentgenol 2003;180:76-77

10. Marsot-Dupuch K, Smoker WR, Grauer W. A rare expression of neural crest disorders: an intrasphenoidal development of the anterior pituitary gland. AJNR Am J Neuroradiol 2004;25:285-88

11. Kizilkilic O, Yalcin O, Yildirim T, et al. Hypothalamic hamartoma associated with a craniopharyngeal canal. AJNR Am J Neuroradiol 2005;26:65-67

12. Megdiche-Bazarbacha $\mathrm{H}$, Ben Hammouda $\mathrm{K}$, Aicha $\mathrm{AB}$, et al. Intras- phenoidal Rathke cleft cyst. AJNR Am J Neuroradiol 2006;27: $1098-1100$

13. Nourbakhsh A, Brown B, Vannemreddy P, et al. Extracranial infrasellar ectopic craniopharyngioma: a case report and review of the literature. Skull Base 2010;20:475-80

14. Arey LB. The craniopharyngeal canal reviewed and reinterpreted. Anat Rec 1950;106:1-16

15. Frieden IJ, Reese V, Cohen D. PHACE syndrome. The association of posterior fossa brain malformations, hemangiomas, arterial anomalies, coarctation of the aorta and cardiac defects, and eye abnormalities. Arch Dermatol 1996;132:307-11

16. Gilbert MS. Some factors influencing the early development of the mammalian hypophysis. Anat Rec 1935;62:337-59

17. Müller F, O'Rahilly R. The development of the human brain from a closed neural tube at stage 13. Anat Embryol 1988;177:203-24

18. Ikeda H, Suzuki J, Sasano N, et al. The development and morphogenesis of the human pituitary gland. Anat Embryol 1988;178: 327-36

19. Kollias SS, Ball WS, Prenger EC. Review of the embryologic development of the pituitary gland and report of a case of hypophyseal duplication detected by MRI. Neuroradiology 1995;37:3-12

20. Sperber GH, Sperber SM, Guttmann GD. Craniofacial Embryogenetics and Development. Shelton, Connecticut: People's Medical Publishing House-USA; 2010:105-13

21. Lowman RM, Robinson F, McAllister WB. The craniopharyngeal canal. Acta Radiol Diagn (Stockh) 1966;5:41-54

22. Wilkerson WW, Cayce LF. Persistent craniopharyngeal canal in a living adult. Laryngoscope 1949;59:381-96

23. Blustajn J, Netchine I, Frédy D, et al. Dysgenesis of the internal carotid artery associated with transsphenoidal encephalocele: a neural crest syndrome? AJNR Am J Neuroradiol 1999;20:1154-57

24. Pinilla-Arias D, Hinojosa J, Esparza J, et al. Recurrent meningitis and persistence of craniopharyngeal canal: case report. Neurocirugia 2009;20:50-53

25. Goldhammer Y, Smith JL. Optic nerve anomalies in basal encephalocele. Arch Ophthalmol 1975;93:115-18

26. Pasquini E, Faustini-Fustini M, Sciarretta V, et al. Ectopic TSH-secreting pituitary adenoma of the vomerosphenoidal junction. Eur J Endocrinol 2003;148:253-57

27. Arndt S, Wiech T, Mader I, et al. Entire infrasellar craniopharyngioma simulating clival chordoma. Otolaryngol Head Neck Surg 2007;137:981-83

28. Benitez WI, Sartor KJ, Angtuaco EJ. Craniopharyngioma presenting as a nasopharyngeal mass: CT and MR findings. J Comput Assist Tomogr 1988;12:1068-72

29. Byrne MN, Sessions DG. Nasopharyngeal craniopharyngioma. Case report and literature review. Ann Otol Rhinol Laryngol 1990; 99:633-39

30. Lewin R, Ruffolo E, Saraceno C. Craniopharyngioma arising in the pharyngeal hypophysis. South Med J 1984;77:1519-23

31. Coppit GL 3rd, Perkins JA, Manning SC. Nasopharyngeal teratomas and dermoids: a review of the literature and case series. Int $J$ Pediatr Otorhinolaryngol 2000;52:219-27

32. Husein OF, Collins M, Kang DR. Neuroglial heterotopia causing neonatal airway obstruction: presentation, management, and literature review. Eur J Pediatr 2008;167:1351-55

33. Manjila S, Miller EA, Vadera S, et al. Duplication of the pituitary gland associated with multiple blastogenesis defects: duplication of the pituitary gland (DPG)-plus syndrome. Case report and review of literature. Surg Neurol Int 2012;3:23 